University of Minnesota Morris Digital Well

University of Minnesota Morris Digital Well

$10-2013$

\title{
Hilary Putnam's Consistency Objection against Wittgenstein's Conventionalism in Mathematics
}

Pieranna Garavaso

University of Minnesota, Morris, garavapf@morris.umn.edu

Follow this and additional works at: https://digitalcommons.morris.umn.edu/philosophy_facpubs

Part of the Logic and Foundations of Mathematics Commons

\section{Recommended Citation}

Garavaso, "Hilary Putnam's Consistency Objection against Wittgenstein's Conventionalism in Mathematics," Philosophia Mathematica, 21(III), (2013): 1-18.

This Article is brought to you for free and open access by the Faculty and Staff Scholarship at University of Minnesota Morris Digital Well. It has been accepted for inclusion in Philosophy Publications by an authorized administrator of University of Minnesota Morris Digital Well. For more information, please contact skulann@morris.umn.edu. 
Hilary Putnam's Consistency Objection against Wittgenstein's Conventionalism in Mathematics

\section{Introduction}

Hilary Putnam first published the so-called consistency objection in $1979 .{ }^{1}$ At that time,

the objection was directed against Ludwig Wittgenstein's conventionalist account of

mathematics. It was not an objection which Putnam raised and then forgot. (It is common to ask

how many Putnams ago a claim was made!) In their Introduction to the revised 1983 Second

Edition of the well-known anthology Philosophy of Mathematics, Hilary Putnam and Paul

Benacerraf raised this objection against all conventionalist accounts of mathematics:

Conventionalists attempt to account for mathematical truth by [reducing it] to the truth of certain conventions (by our fiat) plus the preservation of truth through logical

consequence. ... we should mention one reply to the conventionalist that is not often made but is worth considering:

Everything "true by convention" is supposedly true. But conventions, however wellintentioned, can turn out to be inconsistent. First, their consistency or inconsistency is a mathematical fact..., one that is itself hard to represent as a matter of convention. ... This suggests that our ability to make even axioms "true by convention" is already limited by the (nonconventional) fact of their logical consequences. But might we not make contradictions themselves true by convention? Few so far have been so devoted to conventionalism as to suggest that as a way out. ${ }^{2}$

The thrust of the objection is well contained in this passage; however, I will discuss its 1979

version, because it is better developed and the so-called scenario argument, which supports the

key premise of Putnam's objection, appears only in the earlier version. ${ }^{3}$

\footnotetext{
${ }^{1}$ H. Putnam, “Analyticity and Apriority: Beyond Wittgenstein and Quine," Midwest Studies in Philosophy, Vol. IV, Minneapolis: University of Minnesota Press, 1979, pp. 423-41; reprinted in Putnam, Philosophical Paper. Volume 3, pp. 115-138; quotations in the text are from the reprinted version. Putnam's objection can be seen as a version of Quine's critique of conventionalism in "Truth by Convention," The Ways of Paradox and Other Essays, Cambridge: Harvard University Press, 1976, pp. 77-106. Putnam acknowledges his debt to Quine in P. Benacerraf and H. Putnam, eds., Philosophy of Mathematics. Selected Readings, Second Edition, Cambridge University Press, 1983, p. 23. The so-called scenario argument by which Putnam supports this objection is original with him.

${ }^{2}$ P. Benacerraf and H. Putnam, eds., Philosophy of Mathematics. Selected Readings, p. 23. Putnam seems not to even consider the possibility of logics, like for example paraconsistent logic that "accommodates inconsistency in a sensible manner that treats inconsistent information as informative." (Graham Priest and Koji Tanaka,

"Paraconsistent Logic," Stanford Encyclopedia of Philosophy, last accessed 9/5/2012.

${ }^{3}$ Interestingly enough, there are very few discussions of this objection in the literature: Baker 1984, especially $282-$

84; Garavaso 1988, Humphrey 1991, 1993; and Mühlhölzer forthcoming, pp 19-22.
} 
The main focus of this paper is the consistency objection, not whether Wittgenstein's account of mathematics can meet it. First, I outline the controversy between Wittgenstein and Putnam and state the objection. In the third section, I discuss the scenario argument. Putnam believes that his objection refutes all conventionalist accounts of mathematics. As this wide applicability is not apparent, in the fourth section, I raise the objection against an imaginary axiomatic theory $\mathrm{T}$ similar to Peano arithmetic in all relevant aspects, yet not hindered by unnecessary detail. This reformulation requires a few new symbols, but is worth the effort, for its discussion reveals which accounts are subject to this objection, and which are not. In the end, I argue that a conventionalist can explain the structural features of $\mathrm{T}$ such as its consistency by appealing to the connection between rules of inference and their outcome, and suggest that an analogous conventionalist explanation can be provided for the consistency of Peano arithmetic.

If I am correct, the only conventionalist accounts of mathematics that can meet Putnam's objection are grounded in a conventionalist account of the connection between rules and their outcome. This requires a conventionalist account of what constitutes a logical consequence. Thus, one corollary of the argument of this paper is that a wholly conventionalist account of mathematics requires a conventionalist account of logic. I believe that Wittgenstein's conventionalism extends so far, but a full defense of this claim or of the overall plausibility of any such extended conventionalist account requires another paper.

\section{The Consistency Objection}

Putnam examines a few conventionalist accounts of mathematics attributable to Wittgenstein. Right away he clarifies that he does not share Michael Dummett's portrayal of Wittgenstein's account as a 'full blooded' conventionalism according to which "the logical necessity of any 
statement is always the direct expression of a linguistic convention," as defined in his paper on Wittgenstein's philosophy of mathematics (1959, 329). Instead, Putnam assumes a notion of conventionalism rooted in our forms of life as defended by Barry Stroud who denies that Wittgenstein held a view of mathematical necessity such as Dummett's full blooded conventionalism (1965). By considering objections to each conventionalist account of mathematics attributable to Wittgenstein, Putnam attempts to show "that not even the most sophisticated of these 'Wittgensteinian' views is tenable" (115). Putnam summarizes this most sophisticated view in one claim: "mathematical truth and necessity arise in [elsewhere, from] us."4 By this, he means that "it is human nature and forms of life that explain mathematical truth and necessity." 5 Let us shorten the expression "explained by our nature and forms of life" with "h-explained," and define an explanation that refers just to us, our biology, cultural history, etc., as an "h-explanation." For Putnam, the best of Wittgenstein's conventionalisms can be expressed by the generalization "all mathematical truths are h-explained."

In 1979, Putnam states his objection as follows:

[C]onsider number theory (Peano arithmetic) in any of its standard formalizations. Even if our acceptance of the Peano axioms is just the acceptance of a bunch of meaning

\footnotetext{
${ }^{4}$ H. Putnam, "Analyticity and Apriority: Beyond Wittgenstein and Quine," p. 117; the addition in brackets is mine. Expressions like "due to us," "up to us," etc., are totally uninformative unless it is clear to whom "us" refers. Putnam's clarification of the intended meaning of such expression, quoted in the text, indicates that for him, "us" refers to human beings with the historical and biological development which characterize our species. Interestingly, these expressions are not easily found in Wittgenstein's actual writings.

${ }^{5}$ Ibidem, 117.

${ }^{6}$ It is puzzling that Putnam uses the word 'explain' to characterize Wittgenstein's best account for there are many passages in both the Philosophical Investigations and the Remarks on the Foundations of Mathematics in which Wittgenstein rejects the view that we can justify or explain mathematical truth and/or necessity. For a more extended criticism of Putnam's language in stating Wittgenstein's view, see Mühlhölzer forthcoming :"Nothing could be further from Wittgenstein's intentions than to use concepts like "form of life" in an explanatory function. Wittgensteinian terms like "form of life", "practice" or "language game" are not fit for explanations, and Wittgenstein never used them in such a way." p. 20. I cannot discuss this point adequately here, but I want to stress that I maintain Putnam's wording merely for accuracy not because I believe that Wittgenstein intended his view as providing an explanation or justification for mathematical truth and necessity. For relevant passages in the Philosophical Investigations, see Part I, §§ 5, 71, 87, 109, 126, 209, 211, 496, 654-55; in the Remarks, see: Part I, § 19; Appendix I, § 2; Part III, § 41, 74; Part VI, § 3, 4, 23, 24 (“ask for no reason! ... I can give no reason.”); Part VII, § 40,
} 
determinations ..., still they are not logically arbitrary determinations, for they are, after all, required to be consistent. Our nature, our forms of life, etc., may explain why we accept the Peano axioms as opposed to some other consistent set; but our nature cannot possibly make an inconsistent set of axioms true. And consistency is an objective mathematical fact, not an empirical fact. Thus, there is at least one mathematical fact-namely the consistency of the meaning determinations themselves, whatever these be produced by--which is not explained by our nature or "forms of life" in any intelligible sense. $^{7}$

In the second sentence above, Putnam grants that we may accept the Peano axioms merely as meaning determinations. Of course, this acceptance is h-explained. However, his point is that the truth and thereby the joint consistency of these meaning determinations cannot be hexplained. For, our acceptance could not make all the Peano axioms true if they were inconsistent. As the mathematical truth "The Peano axioms are consistent" is not h-explained, the generalization that Putnam attributes to Wittgenstein is false.

Putnam deals right away with an objection based on what he takes to be a consequence of Wittgenstein's rule following considerations. If there are infinitely many theorems of Peano arithmetic then the claim "Peano arithmetic is consistent" may have no truth-value:

[If] Wittgenstein is right (and I am presently inclined to think that he is), then the statement "there are seven consecutive sevens in the decimal expansion of $\pi$ " may have no truth value .... And similarly, and for the same reason, "Peano Arithmetic is consistent" may have no truth value - for this statement too talks about an infinite sequence (the sequence of all theorems of Peano arithmetic), and the sequence may not really be determinate. ${ }^{8}$

Putnam interprets Wittgenstein's rule following remarks as implying the denial that infinite sequences obtained by the repeated application of a rule are determinate sequences. Two subjects may write the same signs or have the same mental signs for a rule, and yet their practices in following the rule diverge. Since only our practice fixes the correct interpretation of

\footnotetext{
7 “Analyticity and Apriority, 117-118.

8 “Analyticity and Apriority, 119.
} 
a rule and human practice, actual and potential, only extends so far, there is no such thing as the complete and correct interpretation of a rule applied an indefinite number of times.

In reply to this possible counter, Putnam introduces the proposition "Peano arithmetic is $10^{20}$-consistent," i.e., the claim that no contradiction is derivable from the Peano axioms in derivations of $10^{20}$ or fewer symbols. This proposition, Putnam claims, has a determinate truthvalue even on Wittgenstein's account. For, according to Wittgenstein, once a finite sequence of applications of a rule is correctly completed, human practice has determined those applications as the correct ones for that rule. Thus, statements about finite sequences have a determinate truth-value. So, there is a statement whose truth cannot be h-explained and which refutes Wittgenstein's generalization. ${ }^{9}$

Before stating the objection, one point of clarification needs to be made. Putnam grants that Wittgenstein's account of mathematics may h-explain (a) our acceptance of the Peano axioms, but denies that it can also h-explain (b) their consistency. One could interpret this as the denial that the h-explanation which Wittgenstein provides for (a) is an h-explanation for (b). But, this unnecessarily weakens Putnam's objection whose point is not to deny that one and the same story can h-explain both the choice of the axioms and the consistency of Peano arithmetic, but rather that this latter can ever be h-explained. As the claim that (a) is h-explained plays no role in the denial that (b) is h-explained, I state Putnam's objection as follows:

\footnotetext{
${ }^{9}$ Putnam's move to $10^{20}$-consistency may seem unconvincing. First, one may point out that proofs with $10^{20}$ symbols are not any more surveyable than proofs where the number of symbols is not restricted. So, human beings my not know the truth of "Peano arithmetic is $10^{20}$-consistent." Second, Wittgenstein's rule-following considerations imply that any finite number of applications of a rule can be interpreted as fitting different readings of that rule. So, limiting the applications of the rules to $10^{20}$ symbols may still not tell us which interpretation is correct. Both replies seem ineffective. First, Putnam simply needs a statement with a determinate truth-value not one that we actually know. Second, although for Wittgenstein a finite set of application of a rule can support widely divergent ways of applying that rule to a new case, Putnam only needs a finite set of applications regarded as the correct application of a rule so far. Wittgenstein must allow for this assumption as it is the very starting point of his argument about the divergent ways of extending the application of the rule to a new case. The motivation behind Putnam's move, i.e., his assumption that Wittgenstein would deny that a statement about an infinite sequence has a truth value is another matter and I cannot discuss it here.
} 
1. If Wittgenstein's conventionalist account of mathematics is correct, then the $10^{20}$ consistency of mathematics is h-explained.

2. There is no h-explanation for the $10^{20}$-consistency of the Peano axioms.

3. So, Wittgenstein's conventionalist account of mathematics is not correct.

I assume that premise one correctly states Wittgenstein's view: all the features of mathematical systems, e.g., their consistency, and not only the choice of the axioms, are due to us. If this were not Wittgenstein's view, his account would not entirely h-explain mathematics. The key premise is the second. In the next section, I discuss Putnam's major support for it.

\section{The Scenario Argument}

This argument outlines two scenarios of how our dispositions, due to our nature and forms of life, might differently determine the application of a logical rule, e.g., modus ponens. In both scenarios, we consider only derivations of theorems of Peano arithmetic with less than $10^{20}$ symbols. Moreover, the correct application of the rule is determined only by our practice. Putnam's goal is to show that the $10^{20}$-consistency of Peano arithmetic cannot be "due to us."

In the first scenario, one checks the putative proof as follows. For each line whose justification is $a x$, one checks it by determining whether the sentence on that line is an axiom; for each line whose justification is given by the numbers $(\mathrm{n})$ and $(\mathrm{m})$, (and, by our hypothesis, modus ponens), one verifies that the line contains the ponential of the nth and the mth preceding sentences, i.e., the result of the application of modus ponens to the formulas occurring on the nth and mth lines. If any line is " $1=0$ " and each line up to and including that line checks out correct, one declares Peano arithmetic $10^{20}$-inconsistent. In the second scenario, one proceeds as in the first but if on any line " $1=0$ " appears, one deems some line incorrect and adjusts the extension of "the ponential of" so that the proof is incorrect. 
The second scenario describes how our proof-dispositions could affect and explain, in the sense of causally originate, the $10^{20}$-consistency of Peano arithmetic. Here, the absence of a contradiction in the theory is undoubtedly due to us: we so fix the application of modus ponens that if we derived " $1=0$ ", we would exclude it, by deliberate decision, from the set of correct results of the rule. If we determined the extension of "the ponential of" in this way, then the $10^{20}$-consistency of Peano arithmetic could plausibly be regarded as arising from us. ${ }^{10}$ But, Putnam argues, this second scenario is counterfactual. The first scenario depicts our actual behavior and dispositions; here, even if our dispositions determine how we apply any rule, Peano arithmetic comes out $10^{20}$-consistent or -inconsistent independently of our dispositions. ${ }^{11}$ Only if it were the result of our decisions, as described in the second scenario, would the $10^{20}$ consistency of Peano arithmetic be h-explained.

The plausibility of the two scenarios is clearly different: we can see, by analogy to similar past and present cases, that people act like scenario one and not like scenario two. It would also be hard to deny that, if things really were as in scenario two, then the $10^{20}$-consistency of Peano arithmetic would be the direct result of our intervention. This passage from Wittgenstein suggests that he agrees with Putnam on our actual rule following behavior:

"The rules compel me to ...." - this can be said if only for the reason that it is not all a matter of my own will what seems to me to agree with the rule. And that is why it can even happen that I memorize the rules of a board-game and subsequently find out that in

\footnotetext{
10 'Perhaps 'ponential of' is only defined 'finitistically' ... perhaps the extension of 'ponential of' is not fixed in the case of proofs and formulas that are beyond human and machine reach; certainly, in the cases where it is fixed, it is fixed only by our dispositions and not just by the thought-signs in our minds or the representations in our brains; but the $10^{20}$-consistency of Peano arithmetic is still not an artifact of this dispositionally fixed interpretation.' Putnam, Analyticity and Apriority, 120.

${ }^{11}$ It could be objected that since Kripke (1982) has shown that a dispositional account of rule following is unsatisfactory, then any discussion of Putnam's scenario argument in terms of dispositions is moot. This claim misses the point of the consistency objection: even granting Kripke's denial that rule following behavior is dispositional, the consistency objection focuses on the origin of the structural features of the consequence set of a theory, whichever account of the rule-following practice is taken as correct. The contrast between the results of the two scenarios remains the same.
} 
this game whoever starts must win. And it is something like this, when I discover that the rules lead to a contradiction. ${ }^{12}$

In this example, the correct application of the rules of the board game seems to be fixed independent of the result, i.e., that whoever starts must win. Likewise, we may say that the correct application of the rules of inference is fixed by practice independently of the result of applying these rules. This is so even if "I discover that the rules lead to a contradiction."

The two scenarios describe two different reactions to the derivation of an inconsistency. If, while fixing the correct way of applying the rule, we focus on preventing the derivation of contradictions, there is a sense in which the $10^{20}$-consistency of Peano arithmetic is due to us. This is what happens in the second scenario. But if the correct way of obeying a rule is fixed result-independently, i.e., whatever results from applying the rule, as in the first scenario, then such a result cannot be explained by our fixing the correct application of the rule.

Putnam's scenario argument can be expressed as follows:

1. The $10^{20}$-consistency of Peano arithmetic can be h-explained only if our dispositions in fixing the correct way of applying a rule of inference are resultdependent.

2. But, our actual dispositions are result-independent.

3. Thus, there is no h-explanation for the $10^{20}$-consistency of Peano arithmetic. The quote from the Remarks suggests that Wittgenstein accepts the second premise of this argument. I reject the first: there can be an h-explanation for a structural feature of a theory, e.g., its $10^{20}$-consistency, which is compatible with the first scenario. This requires a conventionalist account of the connection between rules and their outcome. So, the scenario argument is unsound and at least one type of conventionalism is not subject to the consistency objection.

\footnotetext{
${ }^{12}$ L. Wittgenstein, Remarks on the Foundations of Mathematics, ed. G.H. von Wright, R. Rhees, G.E.M. Anscombe, trans. G.E.M. Anscombe, Cambridge: The MIT Press, 1983, Part VII, §27, my emphasis.
} 


\section{Theory $\mathrm{T}$}

Much is not essential to Putnam's objection. First, the choice of the number $10^{20}$ is arbitrary. As mentioned before, for the sake of argument, we are assuming that for both Putnam and Wittgenstein, all statements about finite sequences have a determinate truth-value. Second, the feature of the theory for which an h-explanation is required need not be the consistency of Peano Arithmetic. The controversial premise of Putnam's objection, i.e., "There is no hexplanation for the $10^{20}$-consistency of the Peano axioms," is supported by the scenario argument. But the crucial claim of the scenario argument, i.e., "our dispositions in fixing the correct way of applying a rule of inference are result-independent," has nothing to do with consistency or with any other specific feature of Peano Arithmetic. Any structural feature of a theory employing rules of inference could be used to claim that our actual practices in applying rules of inference do not h-explain the resulting features of the relevant theory. Putnam's objection comes down to the claim that the link between a rule and its outcome is independent of any direct human intervention.

Putnam reminds us repeatedly that the consistency of Peano Arithmetic is a "mathematical" fact and that this mathematical fact falsifies Wittgenstein's generalization. Yet, there is no appeal to mathematics in the scenario argument. What is crucial in this argument is the claim that the link between rules and their outcomes cannot be h-explained, i.e., the notion of logical consequence. This suggests that the scope of Putnam's argument is broader than it may seem. The real thrust of his objection is the denial that any wholly conventionalist account of mathematics is given till an analogously conventionalist account of logic is provided. ${ }^{13}$

\footnotetext{
${ }^{13}$ Putnam seems aware of this more general applicability of the consistency objection when he claims that it is a reply to the conventionalist, which is "to some extent parasitic on Quine's reply [as expressed, e.g., in "Truth By Convention]," Putnam and Benacerraf, eds., Philosophy of Mathematics. Selected Readings, Second Edition, 23.
} 
To reveal the broader scope of Putnam's objection, I shall outline a simple imaginary theory $\mathrm{T}$. Theory $\mathrm{T}$ is equivalent to Peano arithmetic in all the aspects relevant to the objection. So, there is no h-explanation for a structural feature of Peano arithmetic such as its $10^{20}$ consistency if and only if there is no h-explanation for an analogous structural feature of T. At the end, I show that an h-explanation of the structural features of $\mathrm{T}$ con be provided which is based on a conventionalist account of the connection between rules of inference and their outcome, and suggest that an analogous h-explanation of the $10^{20}$-consistency of Peano arithmetic can be provided.

Let $\mathrm{T}$ be formulated in the language $\mathrm{L}$. The symbols of $\mathrm{L}$ are ' $*$ ' and ' 0 .' The formulas of $\mathrm{L}$ are any finite sequences of '*' and ' 0 .' The only atomic formula of $\mathrm{L}$ is *. The rules of inference for $\mathrm{L}$ are as follows, for any wff $\alpha$ :

R1: $\quad 0 \alpha$ may be entered on a line if $\alpha$ appears on an earlier line.

R2: $\quad 0 \alpha 0$ may be entered on a line if $\alpha$ appears on an earlier line.

$\beta \vdash \alpha={ }_{\mathrm{df}}$ there is a derivation of $\alpha$ from $\beta$ by R1 and/or R2. $\mathrm{T}=\left\{\alpha: \alpha={ }^{\prime} 0^{*}\right.$ ' or ' $0^{*}$ ' $\left.\vdash \alpha\right\}$. Thus, ' $0 *$ ' is the sole axiom of $T, T_{A x}=\left\{{ }^{\prime} 0^{*}\right\}$. A derivation of $\alpha$ in $T$ is a sequence of wffs $\beta_{1} \beta_{2} \ldots \beta_{n}$ such that $\beta_{\mathrm{n}}=\alpha$, and, for any $j, 1 \leq j \leq n$, either (i) $\beta_{\mathrm{j}}=0^{*}$, or (ii) for some $i<j, \beta_{\mathrm{j}}$ derives from $\beta_{\mathrm{i}}$ by $\mathrm{R} 1$ or R2.

We now define the property of the zero/star-consistency, for short, ZS-consistency. First, call $\alpha$ admissible just in case $\alpha=\beta^{*} \tau$ where $\beta$ is a string of $n>0$ occurrences of ' 0 ' and $\tau$ is a string of $m<n$ occurrences of ' 0 .' Otherwise, $\alpha$ is inadmissible. So, we get the following definition:

$\mathrm{T}$ is ZS-consistent iff ( $\mathrm{T}_{\mathrm{Ax}} \vdash \alpha$ only if $\alpha$ is admissible). 
Let us consider the 11-degree ZS-consistency of T, or the ZS-consistency of the consequence set for $\mathrm{T}_{\mathrm{Ax}}$ in all derivations with at most 11 symbols. I define a derivation of $\alpha$ from $B$ with less than or equal to $\mathrm{n}$ symbols occurring in it as follows:

$\left.\beta\right|_{\leq \mathrm{n}} \alpha=_{\mathrm{df}}$ There is an $\mathrm{n}$-degree derivation of $\alpha$ from $\beta$ by R1 and/or R2. Accordingly, I can introduce the notion of 11-degree ZS-consistency as follows:

$\mathrm{T}$ is 11-degree ZS-consistent iff $\left.\mathrm{T}_{\mathrm{Ax}}\right|_{\leq 11} \alpha$ only if $\alpha$ is admissible.

There is only a finite number of 11-degree derivations and, so, only a finite number of wffs of $\mathrm{L}$ derivable from $\mathrm{T}_{\mathrm{Ax}}$ :

\begin{tabular}{|c|c|c|}
\hline (I) & 1. $0^{*}$ & Axiom \\
\hline & 2. $00 *$ & 1, R1 \\
\hline (II) & 1. $0^{*}$ & Axiom \\
\hline & 2. $00 * 0$ & $1, \mathrm{R} 2$ \\
\hline (III) & 1. $0^{*}$ & Axiom \\
\hline & 2. $00 *$ & 1, R1 \\
\hline & 3. $000^{*}$ & 2, R1 \\
\hline (IV) & 1. $0^{*}$ & Axiom \\
\hline & 2. $00^{*}$ & 1, R1 \\
\hline & 3. $000 * 0$ & $2, \mathrm{R} 2$ \\
\hline (V) & 1. $0^{*}$ & Axiom \\
\hline & 2. $00 * 0$ & 1, R2 \\
\hline & 3. $000 * 0$ & $2, \mathrm{R} 1$ \\
\hline
\end{tabular}

Derivations (I) through (V) fix the correct application of the rules R1 and R2. The consequence

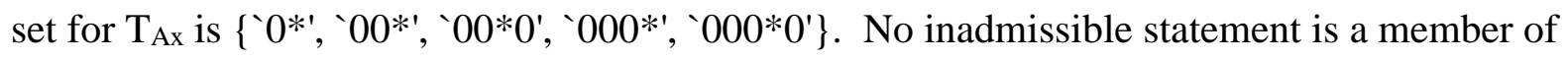
such a set. Hence, T is 11-degree ZS-consistent.

Now, Putnam's objection with regard to T goes as follows:

1. If a conventionalist account of $\mathrm{T}$ is correct, then the 11-degree $\mathrm{ZS}$ consistency of $\mathrm{T}$ is h-explained.

2. There is no h-explanation for the 11-degree ZS consistency of T. 
3. So, there is no conventionalist account of $\mathrm{T}$.

Premise one states the need for an h-explanation of the 11-degree ZS-consistency of T. Again, premise two states the key issue between Putnam and conventionalism. I argue that there is a viable conventionalist account of the 11-degree ZS-consistency of T that successfully refutes Putnam's objection, but before stating it, we need to rule out some unsuccessful replies.

First we must rule out a reply based on Wittgenstein's rule following considerations. One may argue as follows: if there are divergent uses of any rule of inference, each of which appears plausible in some context, then some applications of, e.g., R1 will allow inadmissible wffs. As only our practice fixes the correct application of R1, whether any inadmissible wff is a member of $\mathrm{T}$ depends on us. In this way, the 11-degree ZS-consistency of $\mathrm{T}$ can be h-explained. The following example shows the initial plausibility of this reply. Suppose that, while I apply R1 as in the derivations (I) through (V), above, $\mathrm{S}$ applies it in a bizarre fashion. More precisely, when no '0' occurs in $\alpha$, S adds one before the star, and, when some ' 0 's already occur in $\alpha$, she rearranges the order of the symbols so that in each resulting expression, the first symbol is ' 0 ,' the second is '*,' and then the remaining ' 0 's follow. ${ }^{14}$ Let us call my interpretation of R1 $\left(\mathrm{I}_{1}\right)$ and $\mathrm{S}$ 's interpretation $\left(\mathrm{I}_{2}\right)$. The different consequences appear in the following table:

$\begin{array}{lll} & 0 \alpha \quad\left(\mathrm{I}_{1}\right) & 0 \alpha \\ * & 0^{*} & 0 * \\ 0^{*} & 00^{*} & 0^{*} \\ 00^{*} & 000^{*} & 0^{*} 0 \\ 000^{*} & 0000^{*} & 0 * 00\end{array}$

S's interpretation, which justifies the results in the third column, produces on the fourth row a counterexample to the ZS-consistency of T, i.e., ' $0 * 00$,' while no counterexample appears in the

\footnotetext{
${ }^{14}$ It could be argued that this way of applying R1, unlike mine, is such that $\alpha$ most often denotes two different expressions before and after the arrow in ' $\alpha 0 \alpha$.' This seems correct, but not sufficient to disqualify S's alternative interpretation of R1. For anyone appealing to the rule-following considerations would claim that also the understanding of $\alpha$ in the statement of $\mathrm{R} 1$ is open to interpretation.
} 
column justified by $\left(\mathrm{I}_{1}\right)$. If we applied R1 only once, e.g., to '*,' and we both derived ' 0 *, we might believe that we interpret the rule in the same way. But, as the table shows, at least some future applications of R1 by S will diverge from mine.

Suppose now that $\mathrm{S}$ interprets R2 as I do and that she sets out to derive everything she can from $\mathrm{T}_{\mathrm{Ax}}$ in all 11-degree derivations, Her derivations will look like the following:

(I)

(IV)
1. $0^{*}$
2. $0^{*}$
Axiom
$1, \mathrm{R} 1$
Axiom
$1, \mathrm{R} 2$
2. $00 * 0$
Axiom
1. $0^{*}$
1, R1
2. $0^{*}$
3. $0^{*}$
2, R1
4. $0^{*}$
3, R1
5. $0^{*}$
4, R1

Axiom

1. 0 *

1, R1

2. $0^{*}$

2, R2

Axiom

1. $0 *$

1, R2

2. $00 * 0$

2, R1

While under $\left(\mathrm{I}_{1}\right)$, no inadmissible wff derives from $\mathrm{T}_{\mathrm{Ax}}$, under $\left(\mathrm{I}_{2}\right)$, the consequence set for $\mathrm{T}_{\mathrm{Ax}}$ $\left\{{ }^{\prime} 0 *\right.$, ' $00 * 0,{ }^{\prime}$ ' $0 * 00$ ' $\}$ contains the inadmissible wff ' $0 * 00$.' Which wffs belong to $\mathrm{T}$ is due to which application of R1 is deemed correct. But either S or I determine the correct application of $\mathrm{R} 1$. So, the ZS-consistency of $\mathrm{T}$ is h-explained by our dispositions in applying R1. ${ }^{15}$

\footnotetext{
${ }^{15}$ The point may be raised that S's interpretation of R1 is very implausible and that this threatens the plausibility of this whole reply. However, this point confuses what is really at issue between Putnam and Wittgenstein. Putnam grants Wittgenstein's rule following considerations and these imply that, for any rule, it is always possible to find an interpretation which, no matter how implausible it may seem, would justify a wildly different application of that rule. Putnam's objection focuses on what happens after a rule has been interpreted, not on the issue of how different interpretations of one rule may come about.
} 
However, there is a straightforward objection to this reply. Putnam can point out that, while we determine which rule is denoted by modus ponens, we do not determine the features of the theory derived by modus ponens as meant by us: the rule determines those features. Let $\mathrm{M}_{1}$ and $\mathrm{M}_{2}$ be a pair of finite $10^{20}$ calculators which yield different outputs from at least some identical inputs. When we interpret modus ponens we select either the $\mathrm{M}_{1}$ or the $\mathrm{M}_{2}$ calculations as the correct applications of "ponential of." So, suppose we select $\mathrm{M}_{1}$ and that $\mathrm{M}_{1}$ cranks out no " $1=0$ " for any Peano inputs. Then, under the $\mathrm{M}_{1}$ interpretation of modus ponens, Peano arithmetic is $10^{20}$-consistent. Now, that consistency is due to us in the sense that it is due to the operations of $\mathrm{M}_{1}$, the selection of which is due to us. However, our selection of $\mathrm{M}_{1}$ as our interpretation of how to apply modus ponens does not make it the case that $\mathrm{M}_{1}$ does not yield " $1=0$ " for any Peano input. That $M_{1}$ does not yield " $1=0$ " for any Peano input is not due to us. This is an independent objective fact.

One may be tempted to reply that if we can h-explain why all the formulas in the consequence set of the axiom of $\mathrm{T}$ are admissible we would have h-explained the ZS consistency of T. However, this will not do because we need to h-explain not only why all the formulas that belong to the consequence set of the axiom of $\mathrm{T}$ are admissible but also why only admissible formulas belong to such consequence set. The following analogy clarifies my point. Let A be a student who earns a $\mathrm{C}$ on the midterm, and a $\mathrm{B}$ a student who earns a $\mathrm{C}$ for the course, and suppose that all A's are B's. For each student who earns a C in the midterm, my decisions on the midterm scores directly explain why such a student earns a C. Also, for each student who earns a $\mathrm{C}$ in the course, my decisions on the final grades explain why such a student earns a $\mathrm{C}$ in the course. Yet, the explanations given for the above two facts do not explain the further fact that all A's are B's. This latter fact occurs independently of my decisions concerning who is going to be 
an A or a B; to explain why all A's are B's I need to explain the further fact that a student is an A only if she is also a B. Analogously, to h-explain the 11-degree ZS-consistency of T, we must hexplain why, for each wff $\alpha$ such that $\left.\mathrm{T}_{\mathrm{Ax}}\right|_{\leq 11} \alpha, \alpha$ belongs to $\mathrm{T}$ only if $\alpha$ is admissible.

This discussion brings to light a related point. In seeking to explain why all the members of a set have a certain property, we need to distinguish between narrow and wide scope explanations. In the former, for each member of the set, we explain why that member has that property; in a wide scope explanation, we explain why all the members of the set have that property. My grading analogy clarifies this point. Suppose I review the test of each student who earned a $\mathrm{C}$ in the midterm, and, in each case, what I see in the individual exam makes me decide that that particular student should receive a $\mathrm{C}$ in the course. Here, for each $\mathrm{A}$, a decision of mine explains why A is B. So, this is a narrow explanation. Suppose now that I review the content of the midterm and, given that it covers a crucial part of the course, I decide that whoever earned a $\mathrm{C}$ in it should get a $\mathrm{C}$ in the course. Here, my decision explains why all A's are B's; hence, it is a wide scope explanation. The inadequacy of the mentioned unsuccessful replies shows that the hexplanation for the ZS-consistency of T must be of wide scope.

In the second scenario, Putnam describes what he regards as the only way to h-explain the structural features of a theory. In this scenario, the correct application of R1 and R2 is fixed taking into account the result. I choose, e.g., ' $00^{*}$ ' as deriving from ' 0 ' ' by $\mathrm{R} 1$ because ' $00^{*}$ ' is admissible. If I derive an inadmissible wff, I adapt the criterion of correctness to exclude that particular derivation. Our decisions explain why $\alpha$ is a member of T but also why $\alpha$ belongs to $\mathrm{T}$ only if it is admissible. This seems to constitute a wide scope explanation. ${ }^{16}$ To reject Putnam's

\footnotetext{
${ }^{16}$ It is not fully clear whether Putnam's second scenario provides a wide scope h-explanation. On one side, the decision to accept $\alpha$ as a correct consequence of the application of R1 and R2 is made for each individual $\alpha$ which suggests that the relevant explanation is of narrow scope. On the other side, the decision concerning the individual $\alpha$
} 
objection, the h-explanation of the 11-degree ZS-consistency of T needs to be different from Putnam's second scenario and of wide scope. I argue that there is one such explanation and its illustration concludes my paper.

The following argument supports the denial of the second premise of Putnam's consistency objection with regard to $\mathrm{T}$ :

(R) 1. $\quad \mathrm{T}$ is 11-degree ZS-consistent iff ( $\left.\mathrm{T}_{\mathrm{Ax}}\right|_{\leq 11} \alpha$ only if $\alpha$ is admissible).

2. For any wff $\alpha$ such that $\left.T_{A x}\right|_{\leq 11} \alpha, T_{A x} F_{\# 11} \alpha$ is due to us, i.e., there is an $h-$ explanation of why $\mathrm{T}_{\mathrm{Ax}} \mid \leq 11 \alpha$.

3. For any wff $\alpha$ such that $\mathrm{T}_{\mathrm{Ax}} \mid \leq 11 \alpha$, there is an $\mathrm{h}$-explanation of why $\alpha$ is admissible.

4. There is an h-explanation of why, for any wff $\alpha$ such that $T_{\mathrm{Ax}} t_{\leq 11} \alpha,\left.\mathrm{T}_{\mathrm{Ax}}\right|_{\leq 11} \alpha$ only if $\alpha$ is admissible, i.e., of why all members of $\mathrm{T}$ are admissible.

2. There is an h-explanation of why $\mathrm{T}$ is 11-degree consistent.

Here, several facts are claimed to be h-explained. For each $\alpha$ which is a member of $\mathrm{T}$, there is an h-explanation of (i) why $\alpha$ is a member of T, (ii) why $\alpha$ is admissible, and (iii) why $\alpha$ belongs to $\mathrm{T}$ only if $\alpha$ is admissible. From the definition of the 11-degree ZS-consistency of $\mathrm{T}$ in premise one it follows that there is an h-explanation of this structural feature of $\mathrm{T}$.

(R), if sound, refutes the consistency objection because it promises an h-explanation of the membership of the set of consequences 11-degree derivable from $\mathrm{T}$, of the admissibility of the wffs belonging to $\mathrm{T}_{\mathrm{Ax}}$, and of the structural features of the set of wffs 11-degree derivable from $\mathrm{T}_{\mathrm{Ax}}$. The provided $\mathrm{h}$-explanation is meant to explain all connections between rules and their outcome, thereby including the features of any set of consequences derived from the applications of rules. To understand how one and the same type of h-explanation can explain (iii), above, i.e., why $\alpha$ belongs to $\mathrm{T}$ only if $\alpha$ is admissible, it is useful to review how it explains (ii), above, i.e.., why $\alpha$ is admissible.

is made with the intention to obtain only admissible wffs as consequences which suggests that this same decision can provide a wide explanation. 
A wff is admissible if it satisfies the appropriate definition. For Wittgenstein, definitions like rules have no intrinsic meaning independent of our practice in applying them. We do not discover or come to know the connections between rules and their outcome. We establish them. ' 0 *' is admissible only because we take the definition as deeming ' 0 '”' as admissible, Moreover, there is nothing more ultimate to appeal to, in order to justify our taking ' 0 *' as admissible, than the fact that it strikes us as correct. Hence, our seeing the transition from the rule for admissibility to the statement " $0^{*}$ ' is admissible" as evidently correct provides necessary and sufficient conditions for this statement to follow from the rule. ${ }^{17}$

The statement "' 0 *' is admissible" expresses our seeing ' 0 *' as satisfying the definition of admissibility. But nothing in ' 0 *' or in the definition alone compels the admissibility of ' 0 *.' The truth of " $0 *$ " is admissible" ensues from our regarding as correct a particular way of looking at the definition and the wff, namely that particular way in which the wff strikes us as following from the definition. In taking ' 0 *' as admissible, we further determine the rule for admissibility. There is mutual reinforcement between a rule and its applications. The rule - as a 'signpost' determines the applications, but the applications further determine the rule.

For any wff $\alpha$ 11-degree derivable from $\mathrm{T}_{\mathrm{Ax}}$, the above provides an h-explanation of why $\alpha$ is admissible. There is an analogous h-explanation for the 11-degree ZS-consistency of T. "T is 11-degree ZS-consistent" expresses our natural attitude to look at the consequence set for $\mathrm{T}_{\mathrm{Ax}}$, and at the definition of 11-degree ZS-consistency as such that the set satisfies the conditions required by the definition. $\mathrm{T}$ is 11-degree ZS-consistent if and only if we see all the wffs in $\mathrm{T}$ as admissible. Our looking at the set of wffs in $\mathrm{T}$ and at the definition of ZS-consistency in such a way that we see the wffs in $\mathrm{T}$ as satisfying the definition of admissibility is the only basis for the

\footnotetext{
${ }^{17}$ See Remarks, Part VI, § 5: "The picture shews one, not, of course, anything that happens, but that what ever does happen will allow of being looked at like this." (my emphasis).
} 
11-degree ZS-consistency of T. It is our doing that we see the required structural features of $\mathrm{T}$ as realized. Since it is due to us that the wffs belonging to T satisfy the necessary condition for $\mathrm{T}$ to be 11-degree ZS-consistent, we can h-explain the 11-degree ZS-consistency of T.

The following dilemma may suggest the presence of an ambiguity in the notion of 'seeing' as it occurs in the above h-explanation. Either our seeing is veridical, i.e., if we see something, then there is something, e.g., a pattern, to be seen, or our seeing is not veridical, in which case we may see something even if there is nothing to be seen. If our seeing is veridical, then the structural features of the theory depend on whatever we see rather than on us. If our seeing is not veridical, then any structural features are indeed due to us, but there is no way to distinguish correct from incorrect seeing. The purpose of this paper is to deny Putnam's generalization that no conventionalist account can be given of the structural features of a theory rather that developing or defending the plausibility of any particular conventionalist account. Thus, I cannot here discuss this dilemma extensively. I believe that a conventionalist could choose the second horn of the dilemma and appeal to the surprising agreement in judgments among human beings in distinguishing correct from incorrect accounts. ${ }^{18}$ She would also deny that there are any criteria of correctness independent of human judgments.

For the conventionalist, there is no link between rule and outcome other than our establishing such a link. It is not a matter of choosing between different interpretations of a rule and then, in a second stage, seeing what structural features a theory will have, as a result of our choosing an interpretation or another. For the conventionalist, these two stages are not separate because there are no independent meanings of rules. The fact itself that a certain expression is taken as a rule implying an outcome, is due to us, to our taking that expression as endowed with

\footnotetext{
${ }^{18}$ Wittgenstein repeatedly stresses the remarkable agreement in judgments among human beings, see Philosophical Investigations $\S \S 208-11, \S \S 217-19, \S 242$ and Remarks on the Foundations of Mathematics VI $§ 39$ and $§ 49$.
} 
a normative force with regard to certain outcomes. Thus, there is no significant difference in $\mathrm{h}-$ explaining the admissibility of a wff or the ZS-consistency of a theory. We have a rule and an outcome and they are related if and only if we so look at them that the outcome is seen as fitting the relevant definition.

Of course, there are connections which would seem bizarre to most of us, and others which would seem natural. But, for the conventionalist, this is a matter having to do with the particular natural history which characterizes us as human beings. In actuality, the patterns we see as salient are commonly shared and so we tend to think that they express patterns independent of our own doing. But this is simply habit or custom. ${ }^{19}$ For a conventionalist, these patterns have no independent existence. Yet, if one accepts this h-explanation of the link between all rules and their outcome, then the $\mathrm{ZS}$-consistency of $\mathrm{T}$ is indeed due to us.

This h-explanation does not require that the choice of the wffs which belong to $\mathrm{T}$ be aimed at producing an 11-degree ZS-consistent theory. The 11-degree ZS-consistency of T is due to us, but is not explicitly stipulated while fixing the correct application of the rules. Suppose the situation described by $(\mathrm{R})$ holds. Since the choice of the axioms, of the correct application of the rules of inference, and of the definitions of admissibility and ZS-consistency, is due to us, then, for each $\alpha$, belonging to $\mathrm{T}$, there is an h-explanation of why $\alpha$ belongs to $\mathrm{T}$, of why $\alpha$ is admissible, and of why, for a theory to be 11-degree ZS-consistent, all the wffs 11 degree derivable from the axioms of the theory must satisfy the condition of admissibility. Finally, the set of wffs satisfies this necessary condition if and only if we see it as so doing. Then, the 11-degree ZS-consistency of the theory is due to us. Yet, we did not determine the correct application of the rules so as to obtain an 11-degree ZS-consistent theory. Hence, our

\footnotetext{
19 "I have read a proof - and now I am convinced. - What if I straightway forgot this conviction? For it is a peculiar procedure: I go through the proof and then accept its result. - I mean: this is simply what we $d o$. This is use and custom among us, or a fact of our natural history." Remarks, Part I, § 63; see also Part VI, § 43.
} 
dispositions in fixing the correct application of the rules are result-independent and this account is compatible with the first scenario.

Result-independence is crucial in the debate between Putnam and the conventionalist. The application of a rule can be independent from its outcome in two senses. First, it is resultindependent if it is not affected by the outcome, e,g., it is not determined in view of producing or avoiding the result. In Putnam's second scenario, the correct application of the rule modus ponens is not result-independent in this first sense. Second, the application of a rule is resultindependent if there is no causal connection between the determination of the correct application of the rule and its outcome. The first sense does not require the second. In the above hexplanation of the 11-degree ZS-consistency of T, it is not to avoid an inadmissible wff that we so determine the correct application of the rules. Hence, this application is result-independent in the first sense, but not in the second, because the presence or absence of an inadmissible wff is the causal result of our determination.

If some type of conventionalism like Wittgenstein's is correct, structural features of theories such as the ZS-consistency of T and the consistency of Peano arithmetic are causally dependent on our dispositions and form of life; they are h-explained. The demand that the application of the rules of inference be result-independent in the second sense would beg the question against such a conventionalism. Nonetheless, in such a view, our fixing the correct application of the rules of inference is independent of the result, i.e., is result-independent in the first sense. So, the result causally depends on us, but our determination of the application of rules does not depend on the result.

In conclusion, the examination of the elementary axiomatic theory $\mathrm{T}$ has proved to be fruitful. Theory $\mathrm{T}$ was built to be equivalent to Peano arithmetic in all the respects relevant to 
the disagreement between Putnam and Wittgenstein, but free from unnecessary detail. I then attempted to defend the claim that the structural features of $\mathrm{T}$ are due to us. Given the construed analogy between $\mathrm{T}$ and Peano arithmetic, if Putnam's objection is not successful against the claim concerning the structural features of T, then it is not successful against Wittgenstein's claim that the structural features of Peano arithmetic are due to us. So, I set out to verify the antecedent of this conditional. I reformulated Putnam's consistency objection with regard to $\mathrm{T}$ in its barest form. By quickly ruling out some unsuccessful replies, I was able to illustrate an account of the structural features of $\mathrm{T}$ which constitutes a satisfactory reply to the consistency objection. But by the hypothesis of this paper, if there is an h-explanation for the structural features of $\mathrm{T}$, then there is an h-explanation for the structural features of Peano arithmetic and Putnam's consistency objection can be rejected.

I conclude that the consistency objection is not successful against all conventionalist accounts of mathematics for Putnam's challenge can be met by a conventionalist account of the connection between rules and outcome of rules. Although I do not discuss the plausibility of such a conventionalist view in this paper, I suggest that Wittgenstein's views on following rules of inference may provide an actual example of such a conventionalist account. ${ }^{20}$

\section{REFERENCES}

Baker, L.R., “On the Very Idea of a Form of Life,” Inquiry, 27 (1984): pp. 277-289.

\footnotetext{
${ }^{20}$ The revisions of this paper have been multiple; its first writing was supported by a Graduate School Summer Research Fellowship funded by the University of Minnesota. I presented a shorter version at the 1991 meeting of the Minnesota Philosophical Society and the most recent version at the conference "Perspectives on Wittgenstein's Philosophy of Mathematics" at the University of Zurich (August 2012) and at the $20^{\text {th }}$ Anniversary Conference of SIFA in Alghero (September 2012). I thank Al Casullo, Phil Hugly, Lory Lemke, Ish Haji, Penelope Maddy, Michael Resnik, Stewart Shapiro, Pasquale Frascolla, Felix Mühlhölzer and the audiences at these conferences for helpful comments on previous drafts. I thank my Morris Academic Partner Lincoln Kirkeide for his help with the bibliography and his careful proofreading.
} 
Benacerraf P. and Putnam H., eds., Philosophy of Mathematics. Selected Readings. Second Edition, Cambridge: Cambridge University Press, 1983. Print.

Dummett, M. "Wittgenstein's Philosophy of Mathematics." The Philosophical Review 68.3 (1959): 329.

Garavaso, P. "Wittgenstein's Philosophy of Mathematics: A Reply to Two Objections," The Southern Journal of Philosophy, Vol. 26, 1988, pp. 179-191.

Graham P. and Koji T., "Paraconsistent Logic," Stanford Encyclopedia of Philosophy, last accessed 9/5/2012.

Humphrey, J.A. "Some Objections to Garavaso's Wittgenstein", The Southern Journal of Philosophy, Vol. 29, 1991, pp. 303-327.

Humphrey, J.A. "Some Objections to Putnam's 'Consistency Objection,” Journal of Philosophical Research, Vol 18 (1993): 127-141.

Kripke, S., Wittgestein on Rules and Private Language, Cambridge, Mass.: Harvard University Press, 1982.

Mühlölzer, F. "Putnam, Wittgenstein and the Objectivity of Mathematics," in Randall E. Auxier, ed., The Philosophy of Hilary Putnam (in the Library of Living Philosophers), Open Court, forthcoming.

Putnam, H. "Analyticity and Apriority: Beyond Wittgenstein and Quine." Midwest Studies in Philosophy 4 (1979): 423-41; reprinted in Putnam, Realism and Reason. Philosophical Paper. Volume 3, Cambridge: Cambridge University Press, 1983, pp. 115-138. Print.

Quine, W. V. The Ways of Paradox and Other Essays. Cambridge: Harvard University Press, 1976. Print.

Stroud, B. "Wittgenstein and Logical Necessity." The Philosophical Review 74.4 (1965): 50418. Print.

Wittgenstein, L. Philosophical Investigations. Oxford, UK: Blackwell, 1997. Print.

Wittgenstein, L. Remarks on the Foundations of Mathematics. Cambridge: MIT Press, 1978. Print. 